\title{
Motivation, Satisfaction and Perceived Benefits of Volunteering With the Cardiovascular Health Awareness Program (CHAP): A Survey of French- speaking Volunteers in Quebec
}

Janusz Kaczorowski ( $\nabla_{\text {jausz.kaczorowski@umontreal.ca ) }}$

Department of Family and Emergency Medicine, University of Montreal, and CRCHUM, Montreal, Canada https://orcid.org/0000-0002-8031-4864

Hanaa Moussa

Centre de recherche du CHUM

Marie-Thérèse Lussier

Universite de Montreal

Magali Girard

Centre de recherche du CHUM

\section{Research}

Keywords: Volunteers, Health promotion, Cardiovascular Diseases, Surveys and Questionnaires, Public Housing

Posted Date: September 8th, 2020

DOI: https://doi.org/10.21203/rs.3.rs-72185/v1

License: (a) (i) This work is licensed under a Creative Commons Attribution 4.0 International License.

Read Full License 


\section{Abstract}

Background: Locally recruited and trained volunteers are a vital component of the Cardiovascular Health Awareness Program (CHAP), a community-based cardiovascular disease prevention program that has been implemented across different settings and targeting different populations. As part of expanding and adapting CHAP to new settings, we conducted a pragmatic cluster randomized controlled trial to implement CHAP targeting elderly residents of subsidized social housing buildings in Québec and Ontario. As there is some evidence that the culture of volunteering might be different for English-speakers than their French-speaking counterparts, our objective was to generate a profile of French-speaking CHAP volunteers and to compare it with English-speaking volunteers in Ontario who participated in an earlier community-based CHAP program and with volunteers in Canada as reported in the Statistics Canada's General Social Survey on Giving, Volunteering and Participating (CSGVP).

Methods: A cross-sectional survey was administered online or by telephone to volunteers who participated in at least one CHAP session held in subsidized social housing in Montérégie (Québec, Canada) between March 2018 and June 2019.

Results: The response rate was $79 \%(30 / 38)$, majority were female $(87 \%, 26 / 30)$, retired or semi-retired $(83 \% ; 25 / 30)$, almost two-thirds $(63 \%, 19 / 30)$ had previous work experience in the healthcare field, and $40 \%(12 / 30)$ had more than 10 years of volunteering experience. These characteristics were similar both to those of general profile of volunteers in Canada and to English-speaking volunteers in Ontario who participated in an earlier pharmacy-based CHAP program. The top 5 reasons for volunteering among French-speaking CHAP volunteers were identical to those reported in CSGVP: making a contribution to the community; using one's skills and experience; improving one's sense of well-being or health; exploring one's own strengths; and networking with or meeting people. The majority of French-speaking CHAP volunteers, like their English counterparts in Ontario, reported a high level of satisfaction with their participation in the program, enjoyed their interaction with participants, and gained new knowledge.

Conclusions: French-speaking CHAP volunteers in Quebec share many similarities with English-speaking CHAP volunteers in Ontario and volunteers in Canada in terms of background, motivation, satisfaction and perceived benefits of volunteering.

\section{Background}

Volunteers are recognized as an important human resource for health promotion efforts not only in lowand middle-income countries, but increasingly, in high-income counties such as Canada. Indeed, volunteers have been shown to be effective in supporting behavior modification to help prevent chronic disease among older adults (1), assist in smoking cessation, or improve management of hypertension and diabetes (2). Frequently, they serve as a bridge between the formal health care system, the patient, and community programs and resources $(3,4)$. Volunteers can play a key role in strengthening health 
systems to provide person-centered care that is equitable, culturally appropriate and economically feasible (5).

Older volunteers, in particular, bring a wealth of experience that benefits their communities but also themselves. Volunteering, particularly by the elderly, may contribute to physical, social, and cognitive health and well being (6-10), as well as to an increased quality of life, sense of self-worth and longevity $(10,11)$. The increased social networks, resources, power and prestige, and emotional fulfillment that can be gained through volunteering have been documented as some of the additional benefits (12).

In the province of Quebec in Canada, the culture of volunteering appears to be different from the rest of the country. The 2004, 2007, 2010 and 2013 Canada Survey of Giving, Volunteering and Participating (CSGVP) reported that Quebec is the Canadian province with the least amount of volunteering (13). In $2013,32 \%$ of people aged 15 and older volunteered in Quebec, the lowest rate in the country, compared to the Canadian average of $44 \%$ (13). Furthermore, the average yearly number of hours volunteered in Quebec in 2013 was 123 hours, the lowest in Canada, compared to a national average of 154 hours (13). An analysis of 2012 Statistics Canada data by the Institut de la Statistique du Québec revealed differences in volunteer participation between French-speaking and English-speaking Quebecers (14). The study reported that Quebecers whose language spoken at home is English are more likely to volunteer (47\%) than those whose spoken language at home is French (36\%) (14).

The Cardiovascular Health Awareness Program (CHAP: www.CHAPprogram.ca) is a community-based cardiovascular diseases (CVD) prevention and management program that has been implemented in more than 40 cities and communities across Canada over the past 20 years. It was first implemented in several English-speaking settings in Canada, predominately in the province of Ontario, and subsequently modified and adapted to several different settings and populations, including the French-speaking province of Quebec.

CHAP recruits, trains, and deploys volunteers to assist members of their communities to increase awareness around CVD, monitor their blood pressure and adopt or maintain a healthy lifestyle. Volunteers are a vital component of CHAP as the program relies on locally recruited peer volunteers to: 1) assist program participants with accurate measurement of participants' blood pressure and other physical measures (waist circumference, weight, height and body mass index (BMI)), completing a cardiovascular risk profile, including a questionnaire-based evaluation of risk of developing type 2 diabetes (CANRISK), 2) provide participants with educational messages about lifestyle modifications, and 3) loop back with primary care providers and recommend locally available free or low cost programs and resources. Based on the evidence collected to date, CHAP can identify adults with undiagnosed or uncontrolled hypertension, significantly lower blood pressure (15), optimize drug regimens (16), reduce cardiovascular hospitalization rates (16), and reduce costs to the health care system (17).

In light of the considerable differences regarding culture of volunteering in Quebec, the objective of the current study was to learn more about French speaking Québec volunteers' motivation, satisfaction and perceived benefits of volunteering. We also wanted to generate a profile of Quebecers volunteering for 
CHAP and to compare it with English-speaking volunteers in Ontario who participated in an earlier pharmacy-based CHAP program (18), with volunteers in Canada and in Quebec as reported in Statistics Canada's General Social Survey on Giving, Volunteering and Participating (CSGVP) $(13,14)$, and with the volunteer survey in Quebec conducted by the Réseau de l'Action Bénévole du Québec (RABQ) (19).

\section{Methods}

As part of a pragmatic cluster randomized controlled trial (RCT), CHAP has been implemented in 14 social housing buildings in Montérégie (Quebec, Canada) and the Niagara region (Ontario, Canada) targeting residents aged 55 and over (20). The current study focuses on volunteers from the Quebec arm of the trial. The CHAP program adapted to this specific context was first pilot-tested in four different senior social housing buildings in Montérégie in March and April 2018 and CHAP sessions, as part of the RCT, were offered from September 2018 to June 2019.

In Quebec, 38 peer volunteers have been recruited through advertisements in local newspapers and by collaboration with local Volunteer offices (Montérégie, Québec, Canada). The offices sent out to their registered volunteers information regarding the CHAP and invited them to participate in the project. They conducted interviews with existing volunteers and with the new candidates who responded to advertisements and updated or facilitated criminal background checks, as appropriate.

Volunteers then participated in a 3 hours training session, facilitated by nurses and the research team. Given that the CHAP sessions were offered over several months period, and that volunteer recruitment took place throughout the project, 6 training sessions were held between March 2018 and March 2019. The CHAP volunteer training session included a theoretical section, which consisted of describing the program and its foundations and a second practical section, which simulated a CHAP session. During the training, volunteers were introduced to the CHAP program and explained their role as a volunteer, the risk factors for CVD, the materials used and the procedures to follow during a CHAP session. They were then able to practice blood pressure measurement using an automated device as recommended by Hypertension Canada guidelines, waist circumference, weight, height, and administering the risk of developing diabetes questionnaire. A volunteer guide containing all the information provided during the training was given to volunteers. It was also during this training session that volunteers signed the confidentiality and consent forms.

A cross-sectional survey in the form of an online questionnaire using Google Form or by telephone, depending on the volunteer's preference, was conducted in summer 2019. All volunteers who assisted in at least one CHAP session between March 2018 and June 2019 were eligible to participate.

\section{Survey instrument}

The survey instrument included multiple-choice and short open-ended questions. When applicable, respondents could choose more than one answer for the multiple-choice questions. A first section of the survey included questions about respondents' general volunteer experiences. The question on reasons for 
volunteering were taken from Statistics Canada's General Social Survey - Giving, Volunteering and Participating, 2018 (21). A second section of the survey consisted of specific questions about their reasons for volunteering with CHAP. A third section was about the level of satisfaction with respect to recruitment and training, logistics (schedule, setting up stations, social housing community room, number of volunteers per session, etc.), communication (with research team, nurses, tenants, other volunteers, volunteer office), and the overall volunteering experience with CHAP. A final section of the survey included questions on learnings and the impact of their volunteering experience.

Data were analyzed with SPSS, version 24.0 for Macintosh. Univariate descriptive statistics and frequency distributions were used to describe the data. The study protocol was approved by the scientific and research ethics committee of the Centre de recherche du Centre Hospitalier de l'Université de Montréal (CRCHUM) and all survey participants provided consent.

\section{Results}

\section{Volunteer profile}

Of the total of 38 volunteers eligible and invited to participate in the survey, 30 completed it resulting in a response rate of $79 \%$. The vast majority were female $(26 / 30 ; 87 \%)$, retired or semi-retired $(83 \% ; 25 / 30)$, and almost two-thirds $(19 / 30 ; 63 \%)$ had a previous work experience in the healthcare field. Almost a quarter $(7 / 30 ; 23 \%)$ had less than 1 year of volunteer experience while $40 \%(12 / 30)$ had more than 10 years. On average respondents assisted in 13 of the 70 CHAP sessions offered in Quebec. Although the volunteer offer was open to all adults, most of the volunteers recruited in the program were older and retired. These characteristics were similar both to those of general profile of volunteers in Canada and to English-speaking volunteers in Ontario who participated in an earlier pharmacy-based CHAP program $(13,1820)$. Those aged 55 and over, and females were more likely to volunteer $(13,1921)$ and their involvement was more likely to be related to the healthcare field (13). In the Ontario CHAP program, the average age of volunteers was $64,88 \%$ (35/40) were female, $48 \%(19 / 40)$ reported background in healthcare, and on average they had volunteered for 19.5 years $(18,20)$.

\section{Motivation for volunteering}

Although motivations for volunteering in general were varied, the five most frequently reported reasons were as follows: 1) To make a contribution to one's community; 2) To make the most of one's skills and experience; 3) To improve one's sense of well-being or health; 4) To explore one's own strengths and 5) To network with or meet people (Table 1). The rankings as well as the percentage of respondents selecting these reasons were virtually identical as those reported in the CSGVP survey (13).

Most respondents $(22 / 30,73 \%)$ defined volunteering as a means of helping themselves and others. Onethird $(10 / 30,33 \%)$ described volunteering as a way to donate their time and experience. Nearly one-third $(9 / 30,30 \%)$ volunteered for personal fulfillment, an experience to try out something new, or a way to share with others. While the question on reasons for volunteering were taken from the Statistics Canada's 
General Social Survey - Giving, Volunteering and Participating, 2018 (21), it was not possible to make comparisons as the results of that survey are not yet publicly available.

\section{Volunteering experience and satisfaction with CHAP}

When asked why they chose to volunteer with CHAP, respondents provided a range of answers. After grouping them into categories, the three most frequently stated categories were: 1) It's in the realm of my profession $(40 \% ; 12 / 30)$; 2 ) To support a good cause $(27 \% ; 8 / 30)$; and 3 ) Personal interest in the topic $(20 \%, 6 / 30)$. There were no comparable questions asked to Ontario CHAP volunteers.

In general, the level of French-speaking volunteer satisfaction with CHAP was very high. The vast majority reported being "somewhat satisfied" or "very satisfied" with the recruitment and training, logistics, communication, and overall experience with the program (Figure 1). The open-ended comments made by some respondents included "good teamwork", "good team spirit" and "interesting project". CHAP volunteers involved in an earlier community-wide demonstration project in two mid-sized communities in Ontario also reported a high level of satisfaction with their participation in the program and enjoyed their interactions with seniors at the sessions (18).

\section{Impact of volunteering with CHAP}

The vast majority of respondents $(77 \% ; 23 / 30)$ felt that they learned from their volunteer experience and that it met their expectations $(93 \% ; 28 / 30)$. When asked about what they gained from their CHAP volunteer experience, respondents indicated several items from the options list provided. Each of the response options was selected by at least one-third of respondents with the exception of "power and prestige" (Table 2). The most frequently selected item, reported by $63 \%(19 / 30)$ of respondents, was "a better understanding of cardiovascular health". The five respondents who selected the option "others" (Table 2) specified it by adding: "better understanding and empathy for people living in social housing", "feeling useful and contributing to the program", " there are many sick people, especially among the elderly", "seeing people and feeling useful to the community" and "beautiful human experience". The English-speaking volunteers in Ontario reported that they felt confident in their role and thought that their contribution was important to the community and rewarding. They also reported becoming more informed about heart health and felt that CHAP was a worthwhile program (mean scores $>4.7$ on a 5 point Likert scales) (18).

\section{Discussion}

Our objective was to draw a portrait of French-speaking CHAP volunteers in Quebec and to compare our results with existing survey data. For this reason and despite the small number of volunteers involved, we chose to use a survey rather than focus groups or individual interviews, which had already been conducted in the past with CHAP volunteers in order to obtain their feedback and make changes to the program $(18,22)$. 
CHAP volunteers in the Montérégie region were mostly retired or semi-retired women who had worked in the healthcare field before and had several years of volunteering experience. This is similar to Englishspeaking CHAP volunteers in Ontario and volunteers in Canada and in Quebec in general. Namely, that those aged 55 and over, females, and people who worked in healthcare are more likely to volunteer $(13$, $18,19)$. The five most frequent reasons for volunteering reported by the French-speaking CHAP volunteers were virtually identical as those reported in the CSGVP survey (13).

Most CHAP volunteers in Quebec defined volunteering as means of offering help, sharing their time and experience which contributed to their sense of fulfillment. While the data collected by the Réseau de I'Action Bénévole du Québec (RABQ) showed that helping was not the top reason for volunteering in Quebec (19), the remaining reasons were very similar and included making a contribution to their community and to use their skills and experience (19). The RABQ study reported that incentives for volunteers in Quebec to get involved were mainly pleasure or interest in a particular activity or cause, the opportunity to socialize, exchange and develop a sense of belonging to a group with whom one shares a common interest, and the realization of a social project or civic cause that was dear to them (19). Similarly, the CHAP volunteers in Ontario reported that they were contributing to the community and that their contribution was important and rewarding (18).

A recent study on the involvement of French-speaking CHAP volunteers revealed that engaging volunteers in the research process was useful to gain important and unique insight on areas of improvement of the research protocol and how to better address needs of CHAP participants (22). The volunteers' feedback led to several adaptations to the research protocol and procedures of CHAP sessions.

The level of satisfaction of CHAP volunteers in Quebec was very high, as was the case with CHAP volunteers in Ontario. In addition to making a contribution to the community, in this case a vulnerable senior population, volunteers also benefited from the experience. The majority reported having learned from their involvement in the program and felt the program met their expectations. Both French-speaking volunteers in Quebec and Ontario CHAP volunteers reported having a better knowledge of cardiovascular health as a result of their involvement with CHAP.

\section{Conclusions}

This study profiled CHAP volunteers involved in an RCT in social housing in the Montérégie region of Quebec, Canada. Although the rates of volunteering and average annual hours of involvement are the lowest in Canada in the province of Quebec (13), the profile of French-speaking CHAP volunteers in Quebec share many similarities with English-speaking CHAP volunteers in Ontario and volunteers in Canada in general in terms of background, motivation, satisfaction and perceived benefits of volunteering.

\section{List Of Abbreviations}


BMl: Body Mass Index

CANRISK: Canadian Diabetes Risk Questionnaire

CHAP: Cardiovascular Health Awareness Program

CSGVP: Canada Survey of Giving, Volunteering and Participating

CVD: Cardiovascular diseases

RABQ: Réseau de l'Action Bénévole du Québec

RCT: Randomized controlled trial

\section{Declarations}

Ethics approval and consent to participate

The study protocol was reviewed and approved by the scientific and research ethics committee of the Centre de recherche du Centre Hospitalier de l'Université de Montréal (CRCHUM) and all survey participants provided written consent.

Consent for publication

Not applicable.

Availability of data and materials

The datasets used and/or analysed during the current study are available from the corresponding author (JK) on reasonable request.

Competing interests

The authors declare that they have no competing interests.

\section{Funding}

This project was supported by a grant from the Canadian Institute of Health Research. The funding agency had no role in study design, data collection and analysis, decision to publish, or preparation of the manuscript.

\section{Authors' contributions}

JK, MTL, and MG conceptualized the study protocol and its implementation. HM carried out analyses and drafted the initial manuscript, and JK reviewed and revised the manuscript. All authors assisted with data 
interpretation and synthesis and reviewed and revised the manuscript. JK, HM, MTL, and MG read and approved the final manuscript as submitted and agreed to be accountable for all aspects of the work.

\section{Acknowledgements}

The authors would like to express a special thanks to all CHAP volunteers and the Centre d'action bénévole de la Montérégie.

\section{References}

1. Webel AR, Okonsky J, Trompeta J, Holzemer WL. A systematic review of the effectiveness of peerbased interventions on health-related behaviors in adults. Am J Public Health. 2010;100:247-53.

2. Jeet G, Thakur JS, Prinja S, Singh M. Community health workers for non-communicable diseases prevention and control in developing countries: Evidence and implications. PloS one. 2017;12(7):e0180640-e.

3. Jandorf L, Fatone A, Borker PV et al. Creating alliances to improve cancer prevention and detection among urban medically underserved minority groups. The East Harlem Partnership for Cancer Awareness. Cancer 2006;107(8 Suppl): 2043-2051.

4. Hale WD, Bennett RG, Oslos NR, Cochran CD, Burton JR. Project REACH: A program to train community-based lay health educators. The Gerontologist 1997;37(5):683-687.

5. Scott K, Beckham SW, Gross M, Pariyo G, Rao KD, Cometto G, et al. What do we know about community-based health worker programs? A systematic review of existing reviews on community health workers. Human Resources for Health. 2018;16(1):39.

6. Chiao C. Beyond health care: Volunteer work, social participation, and late-life general cognitive status in Taiwan. Soc Sci Med. 2019;229:154-60.

7. Fried LP, Carlson MC, Freedman M, Frick KD, Glass TA, Hill J, et al. A social model for health promotion for an aging population: initial evidence on the Experience Corps model. J Urban Health. 2004;81(1):64-78.

8. Konrath S, Fuhrel-Forbis A, Lou A, Brown S. Motives for volunteering are associated with mortality risk in older adults. Health Psychol. 2012;31(1):87-96.

9. Sneed RS, Cohen S. A prospective study of volunteerism and hypertension risk in older adults. Psychol Aging. 2013;28(2):578-86.

10. Morrow-Howell N, Hinterlong J, Rozario PA, Tang F. Effects of Volunteering on the Well-Being of Older Adults. The Journals of Gerontology: Series B. 2003;58(3):S137-S45.

11. Caro FG, Bass SA. Receptivity to Volunteering in the Immediate Postretirement Period. Journal of Applied Gerontology. 1997;16(4):427-41.

12. Musick MA, Herzog AR, House JS. Volunteering and mortality among older adults: findings from a national sample. J Gerontol B Psychol Sci Soc Sci. 1999;54(3):S173-80. 
13. Turcotte M. Volunteering and charitable giving in Canada. Statistics Canada-Statistique Canada; 2015.

14. Nanhou V, Desrosiers H, Ducharme A. Portrait des bénévoles de 16 à 65 ans au Québec. Portraits et trajectoires-Institut de la statistique du Québec. 2017;22:1-28.

15. Ye C, Foster G, Kaczorowski J, Chambers L, Angeles R, Marzanek-Lefebvre F, et al. The impact of a cardiovascular health awareness program (CHAP) on reducing blood pressure: A prospective cohort study. BMC public health. 2013;13:1230.

16. Kaczorowski J, Chambers LW, Dolovich L, Paterson JM, Karwalajtys T, Gierman T, et al. Improving cardiovascular health at population level: 39 community cluster randomised trial of Cardiovascular Health Awareness Program (CHAP). Bmj. 2011;342:d442.

17. Goeree R, von Keyserlingk C, Burke N, He J, Kaczorowski J, Chambers L, et al. Economic appraisal of a community-wide cardiovascular health awareness program. Value Health. 2013;16(1):39-45.

18. Karwalajtys T, McDonough B, Hall H, Guirguis-Younger M, Chambers LW, Kaczorowski J, et al. Development of the volunteer peer educator role in a community Cardiovascular Health Awareness Program (CHAP): a process evaluation in two communities. J Community Health. 2009;34(4):336-45.

19. Fournier M. Portrait des Bénévoles et du Bénévolat. Réseau de l'Action Bénévole du Québec; 2018.

20. Agarwal G, Girard M, Angeles R, Pirrie M, Lussier M-T, Marzanek F, et al. Design and rationale for a pragmatic cluster randomized trial of the Cardiovascular Health Awareness Program (CHAP) for social housing residents in Ontario and Quebec, Canada. Trials. 2019;20(1):760.

21. General Social Survey - Giving, Volunteering and Participating, 2018 (Main), Statistics Canada. Available at: https://www23.statcan.gc.ca/imdb/p3Instr.pl?

Function=assemblelnstr\&lang=en\&ltem_Id=1183690. Accessed on July 20, 2020.

22. Lussier M-T, Kaczorowski J, Girard M, Arpin E. Volunteer engagement to inform research on cardiovascular health awareness, Canada. Health Promotion International. 2020.

\section{Tables}

Table 1: Leading reason for volunteering - CHAP and CSGVP.

\begin{tabular}{|lll|}
\hline Leading reason for volunteering, & $\begin{array}{l}\text { CHAP }(\mathbf{n = 3 0 )} \\
\%(\text { rank) }\end{array}$ & $\begin{array}{l}\text { CSGVP 2013 } \\
\%(\text { rank) }\end{array}$ \\
\hline To make a contribution to the community & $83(1)$ & $93(1)$ \\
\hline To use your skills and experience & $77(2)$ & $77(2)$ \\
\hline To improve sense of well-being or health & $53(3)$ & $52(3)$ \\
\hline To explore your own strengths & $50(4)$ & $49(4)$ \\
\hline To network with or meet people & $47(5)$ & $47(5)$ \\
\hline
\end{tabular}


Table 2: What volunteers have gained from their volunteer experience with CHAP $(n=30)$

\begin{tabular}{|ll|}
\hline & $\mathbf{n}(\%)$ \\
\hline A better understanding of cardiovascular health & $19(63)$ \\
\hline Access to resources & $15(50)$ \\
\hline Emotional fulfillment & $14(47)$ \\
\hline An extended social network & $10(33)$ \\
\hline Power and prestige & $10(33)$ \\
\hline Others & $1(3)$ \\
\hline
\end{tabular}

Figures 


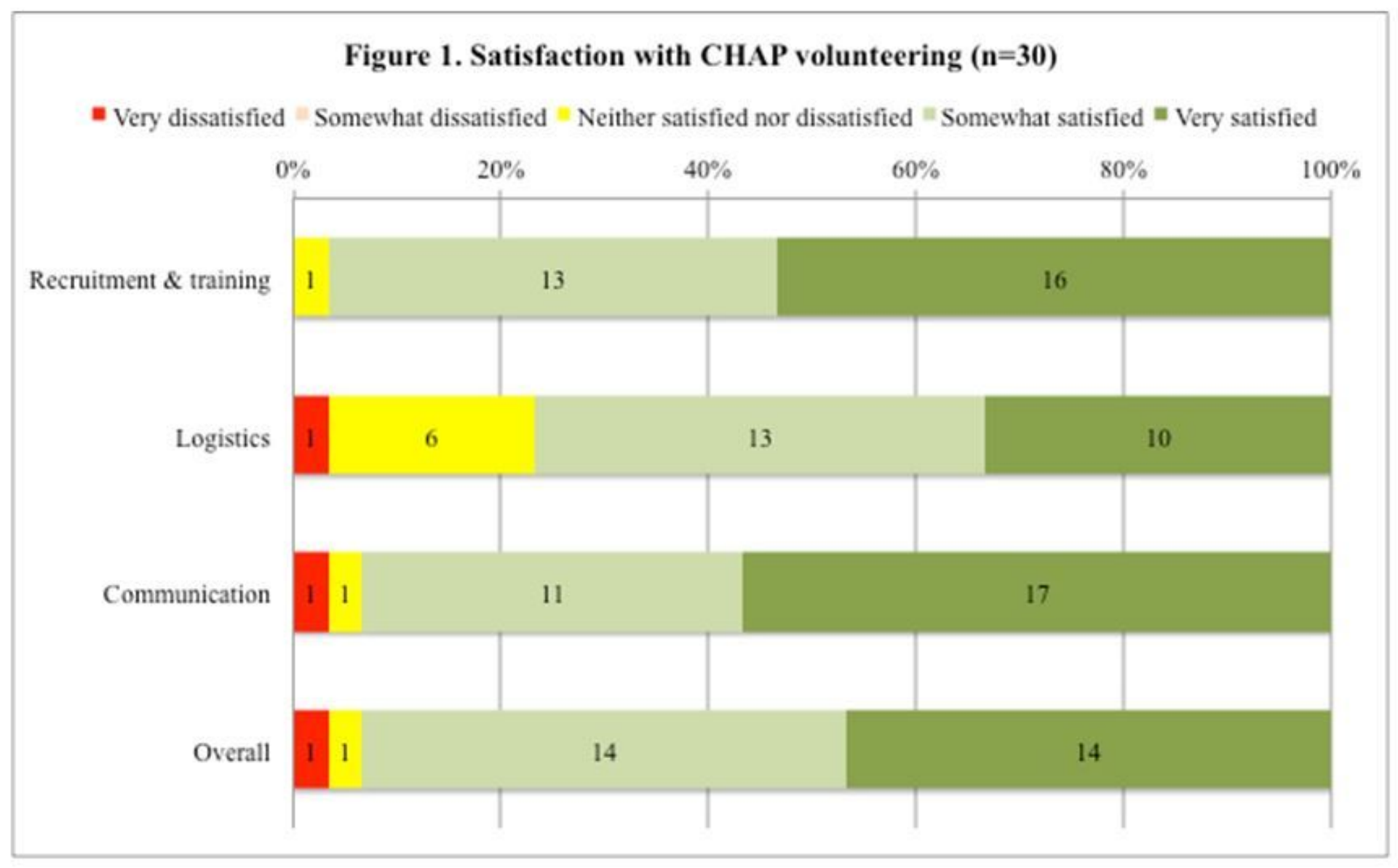

Figure 1

Satisfaction with CHAP volunteering 